$$
\begin{aligned}
& -34\left(p^{6} r^{4}+q^{6} r^{2}\right)-292\left(q^{3} r^{4}+p^{3} r^{5}\right) \\
& +p^{2} q^{8}+p^{8} q^{2} r^{7} \\
& +530\left(p^{4} q r^{4}+p q^{4} r^{3}\right)+50\left(p^{7} q^{3}+p q^{7} r\right) \\
& -4\left(q^{9}+p^{9} r^{3}\right)-12\left(p^{6} q^{3} r^{2}+p^{3} q^{6} r\right)
\end{aligned}
$$

in which $p, q, r$ in terms of the homogeneous variables are

$$
\begin{gathered}
p=Z_{1}+Z_{2}+Z_{3}, q=Z_{1} Z_{2}+Z_{1} Z_{3}+Z_{2} Z_{3}, \\
r=Z_{1} Z_{2} Z_{3} .
\end{gathered}
$$

It is to be noted that $P$ itself is not expressible in terms of $p, q, r$, but

$$
p=Z_{1} Z_{2} Z_{3}\left(Z_{1}-Z_{2}\right)\left(Z_{1}-Z_{3}\right)\left(Z_{2}-Z_{3}\right) \text {. }
$$

As a remarkable coincidence it was found that the three invariants of the complete form-system of the binary quintic form, when written in terms of a fundamental system of two cross-ratios of the roots, are precisely these forms $A, P^{2}, C$, when similarly expressed in terms of the crossratios. It is shown that $A, P^{2}$ and $C$ are the complete form-system of our quadratic group, $G_{120}$, by a series of theorems of which the most important are the following: (1) An invariant under a quadratic operator must be a fraction whose numerator and denominator throw off a common factor in the $Z$ 's. (2) The numerator and denominator of an invariant fraction must be absolute or relative invariants under the linear subgroup and hence rational integral functions of the known invariants in its complete form-system. (3) There can be no invariant fraction whose numerator and denominator are of odd degree or of unequal degree. (4) The most general invariant form suitable for numerator or denominator of an invariant fraction under $G_{120}$ is of degree $6 n$ and throws off the factor $r^{2 n}\left(r=Z_{1} Z_{2} Z_{3}\right)$ under the quadratic generator; $Z_{1}^{\prime}: Z_{2}^{\prime}: Z_{3}^{\prime}=Z_{2} Z_{3}: Z_{1} Z_{3}: Z_{1} Z_{2}$. (5) The most general invariant form under $G_{120}(a)$ is of the form $R_{6 n}=P^{2 \mu} R_{6(n-2 \mu)}$, where $\mu=0$ or a positive integer and $R_{6(n-2 \mu)}$ contains no factor of $P$; and $(b)$ has at each of the four critical points a multiple point of order $2(n+\mu)$. (6) If $\alpha$ and $\beta$ are two such ternary forms having the binary forms $a$ and $b$, of degree $\lambda$ and $\mu$, respectively, as tangential quantics at one of the critical points, then $\alpha \beta$ has $a b$ as its tangential quantic at the same multiple point, and $\alpha+\beta$ has $a, b$ or $a+b$ according as $\lambda$ is less than, greater than or equal to $\mu$. (7) No ternary form can have a binary tangential quantic at any critical point of odd degree in either or both of the cubic invariants belonging to the dihedron subgroup which leaves the critical point fixed. (8) Two reduced ternary forms of the same degree which have the same tangential quantic at any critical point can differ only in such terms as involve $P^{2}$ as a factor.

By means of these theorems it is then shown, by a process of successive reduction, that the most general invariant form under $G_{120}$ is expressible as a rational integral function of $A, P^{2}, C$, and thus a system of fundamental forms is established in terms of which all invariant fractions under the quadratic group can be expressed. The above forms are absolute invariants. The only relative invariant fractions are those expressible in terms of $A, P$ and $C$, which are invariant except for change of sign.

THE CONFERENCE OF SCIENCE TEACHERS IN THE TRANS-MISSISSIPPI EDUCATIONAL CONVENTION.

A FEw months ago the undersigned was requested by the program committee to arrange a series of conferences of science teachers in connection with the Trans-Mississippi Educational Convention, to be held in Omaha, June 28th, 29th and 30th. As a result there were held seven conferences, namely, in Chemistry, Physics, Astronomy, Botany, Zoology, Geography and Geology, occupying the afternoon sessions of the 29th and 30th. The following abstracts of the principal papers will give some idea of these meetings. The attendance was not large, 
being from thirty-five to forty, which was to be expected, since there were conferences upon twelve other lines of study in other places of meeting in the city at the same time.

Professor C. S. Palmer, of the University of Colorado, spoke on the teaching of chemistry. He limited the scope of his remarks to the high and preparatory schools, and noted that only one-fourth of the time is asked for natural science, the other threefourths involving mathematics, language, (English and Latin), literature and history. He noted that there are two kinds of chemistry to be considered: one, that suited to the one-year course of the average school; and the other a much more concentrated and specialized sort, suited to the newer standards now coming to recognition. He gave the chief arguments for natural science specialization in the high school, defending it against the accusation of narrowness, and emphasizing its disciplinary value.

In view of this he emphasized the value of the qualitative proof in chemistry, leavthe quantitative proof for the college course in the main.

(His remarks will be found in the fuller form in a forthcoming article in the School Review, with special reference to a discussion and defence of specialization in natural science in secondary schools.)

Instructor H. V. Kepner, of the Denver Manual Training High School, led this discussion, emphasizing the contention of Professor Palmer.

In the physics conference a paper on ' Graphical Algebra for High Schools' was presented by Professor F. E. Nipher, of St. Louis. This paper was distributed in printed form. It was really an elementary text giving the author's idea of what should be undertaken in connection with instruction in algebra as now taught

Equations based on simple physical con- ditions were written, and the physical significance of each equation was pointed out. The equations were each represented graphically by curves, lines or surfaces. The geometrical meaning of making equations simultaneous and determining the values of the 'unknown quantities' by elimination was fully shown. The paper will be published as a text-book by Henry Holt \& Co.

In the discussion by President C. L. Mees, of the Rose Polytechnic Institute; Professor H. T. Eddy, of the University of Minnesota, and Professor B. E. Moore, of the University of Nebraska, the importance of graphical methods of representing results in Physics was emphasized, and the high schools were urged to give more attention to training their pupils in graphical conceptions.

Professor H. A. Howe, of the University of Denver, presented a paper on 'Astronomy,' in which he said that instruction in astronomy may well be a part of nature study in the four grammar grades. The moon's phases and motion, the seasonal change of the sun's place in the heavens, the motion of bright planets among the stars, and the appearance and changes of position of a few constellations, may be familiarized by observation.

In the high school elementary descriptive astronomy may best be taught in the fourth year; accurate observation of the diurnal revolution of the heavens, and the learning of several constellations, may well precede any work with the text-book. Observation of the heavens and the development of principles and facts by judicious questions should be strongly pushed; memoriter work should be reduced to the lowest limit consistent with a fair general knowledge of the subject.

Collegiate descriptive astronomy is simply more thorough than high school work, and may involve more mathematics. Collegiate mathematical astronomy is most 
interesting and profitable where simple instruments for time-taking and for micrometric measurements are used by the students; where instruments are not available the simpler problems of spherical astronomy may be studied.

Work in celestial mechanics and original investigations along lines of practical astronomy and astrophysics must be done chiefly in post-graduate courses.

In the conference on botany Professor Macbride, of the University of Iowa, argued that the special function of the schools was to give instruction to the people, primarily along practical lines. Botany is an eminently practical science and should reach the largest possible number of our people. To this end botany teaching should not be relegated to the high school exclusively, but simple phases of the subject should be presented to the children of the schools universally. The later methods of presenting the subject, while no doubt logical, have been in large measure unfortunate and without practical value. They have proceeded upon the notion that without microscope and laboratory botany cannot be taught; and, where introduced, they have too often left pupils without any true conception of botanical science, without any knowledge whatever of the commoner forms of vegetation with which every intelligent person, not to say educated person, ought to be familiar. The older methods were faulty because they were so largely text-book methods, but they had the great advantage of using familiar material, of working from the known to the unknown. While unwilling to discourage research-work anywhere, yet, it was argued, such work belongs rather to the universities. In short, public schools of all grades should under. stand well their mission, which is popular rather than technical education.

Professor J. H. Powers, of Doane College, and Professor C. E. Bessey, of the Univer- sity of Nebraska, thought that the difficul ${ }^{-}$ ties in regard to the equipment and management of laboratories in the high schools were not as great as Professcr Macbride appeared to believe, and urged the laboratory method as more helpful in high school work.

In the conference in zoology Professor H. B. Ward, of the University of Nebraska, said that since the time of Agassiz no one can question the educational value of zoology nor its consequent right to a place in the high school curriculum. As a natural development of the nature study in the lower grades, it may well come early in the course, preceded at most by a year of general science and physiography, and closely connected with work in botany.

At least half of the time should be devoted to laboratory work, where the observations made by the student independently should be recorded in careful notes and accurate drawings.

To bring the pupils in touch with nature, field excursions should form an integral part of every course. They also yield a desirable collection of objects representing the life of the vicinity and form the nucleus of a school museum which has no reason for existence other than as a working collection.

Constant use should thus be made of the three laboratories: The school room, the museum and the great laboratory of nature. Finally, students should be encouraged to read the travels of great naturalists and thus, through the eyes of Wallace, Darwin, or Agassiz come in contact with the great world of living things.

Professor J. H. Powers, of Doane College, in discussing the paper, spoke earnestly in favor of zoological training, emphasizing the value of laboratory and field work. Professor H. W. Norris, of Iowa College, in continuation insisted that the end sought in zoological instruction is not so much an acquaintance with interesting and profitable 
facts as such, but rather the cultivation of an ability to discriminate between the essential and non-essential, and to appreciate the fundamental relationships existing between the various groups in the animal kingdom.

Professor Erasmus Haworth, of the University of Kansas, in the geographical conference dwelt upon the present unscientific treatment of geography in the schools, and discussed at some length the need of a reform in both method and subject-matter. Much of what is given under the name of geography is not geography at all, although it may well be admitted that this non-geographical matter is often quite interesting and in some cases possibly useful. Often, however, there is a large amount of gross and inexcusable error, along with much irrelevant and unimportant matter to be found in the text-books.

A lively discussion followed, in which the current text-books on geography were described as being nearly all bad.

In the conference in geology Professor Todd, of the University of South Dakota, read a paper on 'Geology as a Factor in Education.' He enumerated the various advantages for culture which the study presents. It is equal to any other natural science in its cultivation of the power of observation, while no other has its material so universally accessible and so permanently available. Other sciences often required the use of costly instruments and collections. It cultivates reasoning, especially inductive reasoning, and gives practice in the 'scientific method.' Because its problems deal with all degrees of accuracy and probability it especially fits one for the problems of actual life. No other science can equal it in its cultivation of the imagination and in teaching its legitimate bounds. Because of its tangible data and the importance of its conclusions to related subjects of wide interest, it more than others cultivates skill in clear and accurate description. It strengthens the moral nature by instilling love for truth, by revealing the marks of an intelligent purpose in the cosmos and by teaching man's humble position, yet great power if he learns to work with Nature. It discovers a healthful and rational recreation. It brings one in touch with many of the great economic problems of society. He concluded that its clearer and simpler principles, which have been grouped under Physiography, should be required in the high school, as was approved by the 'Committee of Ten,' and that more might be offered at that stage as an elective. He presented reasons for deferring the more thorough pursuit of the science until after fair acquaintance with chemistry, physics, botany and zoology, and then at least a year of geology (including mineralogy) should be required of all candidates for the degrees of B.Sc., C.E. and M. E., while it should be accessible as an elective to all.

Professor E. H. Barbour, of the University of Nebraska, and Professor Erasmus. Haworth, of the University of Kansas, followed in a discussion which emphasized the culture value of geology in the public schools, no other culture excelling it in the cultivation of the power of close observation.

$$
\begin{aligned}
& \text { Charles E. Bessey, } \\
& \text { Chairman of Science Conferences. }
\end{aligned}
$$

\section{CURRENT NOTES ON ANTHROPOLOGY.}

THE BEGINNINGS OF MIND.

A pleasant address on this subject by Dr. Julius Donath, of Budapest, is worth mentioning. It presents in an easy style the accepted principles of modern psychology, and in their light traces the growth of mind in relation to cerebral action in the development of the infant and child and in the species, as exhibited in the contrasts between savage and civilized modes of 